\title{
Silicon strip staves and petals for the ATLAS Upgrade tracker of the HL-LHC
}

\author{
Sergio Díez, on behalf of the ATLAS Collaboration \\ Lawrence Berkeley National Laboratory, 1 Cyclotron Rd, Berkeley (CA), 94720, USA. Phone: (+1) 5104952461. E-mail: sdiezcornell@lbl.gov
}

\begin{abstract}
This paper describes the baseline integration structures for the silicon strip sensors to be used in the ATLAS detector for the Phase-II upgrade of the Large Hadron Collider (LHC) machine, the so-called High Luminosity LHC (HL-LHC). Highly modular structures have been developed for the integration of the silicon strips sensors, readout electronics, cooling, and support structures, called 'staves' for the barrel region and 'petals' for the end-caps of the ATLAS strips tracker. This work describes the status of the current prototypes, the building procedure, designed for mass production even at a prototyping stage, and their electrical performances.
\end{abstract}

Keywords: HL-LHC, ATLAS Upgrade, silicon strip sensors, staves, petals

\section{Introduction}

The LHC experiment at CERN is extending the frontiers of particle physics with its unprecedented high energy and luminosity. Looking towards the future, studies are underway to increase the capabilities of the LHC machine and its detectors, in order to further extend their sensitivity to new physics. With that purpose, a series of experimental upgrades are planned in the following years: phase-0, in 2014, phase-I, in 2017-1018, and phase-II, in 2022. Phase-II upgrade, the so-called High Luminosity LHC (HL-LHC), is foreseen to increase the instantaneous luminosity of the machine by a factor of ten with respect to the current LHC, up to a integrated luminosity of $3000 \mathrm{fb}^{-1}$ [1]. This luminosity increase poses numerous technological challenges to be faced by the LHC detectors: The new detectors must be faster, more highly segmented, cover more area, be more resistant to radiation, and require much greater power delivery to the front-end systems. At the same time, they cannot introduce excess material which could undermine performance. For these reasons, the current ATLAS tracker, the so-called Inner Detector (ID), will be replaced by an all-silicon upgraded tracker. It will consist of several layers of particle detectors. Figure 1 shows a sketch of the latest layout for the ATLAS silicon tracker. The four innermost layers of the central region (barrel) will consist of silicon pixel sensors and the five external layers will consist of silicon strip sensors, with six pixel and five strip disks in each forward region (end-caps). Simulations predict a maximum fluence, including a safety factor of 2 , equal to $1.2 \times 10^{15} n_{e q} / \mathrm{cm}^{2}$ for the innermost layers of the strip barrel [2].

This study is focused in the baseline design of the highly modular structures which are being studied and developed to host the silicon strip sensors, called 'staves' in the barrel and 'petals' in the end-caps. Figure 2 shows a sketch of both integration structures. Both structures follow the same conceptual design [3]. They consist of single-sided strip modules, glued onto laminated kapton-copper bus tapes at the two sides of a low mass carbon-based core structure with embedded titanium cooling pipes. Readout, control, and power electronics are hosted in kapton flex hybrids, glued directly to the silicon sensors with electronic grade epoxy. A stave hosts 12 identical sensor modules per side, one side having axial strips and the other a small stereo angle. Both sides of a petal are identical, hosting 9 sensor modules of 6 different geometries. A small stereo angle is built between both petal sides, resulting in a u-v topology. The development of the stave and petal prototypes is an effort driven by a collaboration of more than 20 institutes worldwide [3,4]. This paper summarizes some of the latest progress of this collaboration. An alternative integration concept for the ATLAS Upgrade strips tracker is the so-called 'supermodule' concept. Detailed studies for this approach are published elsewhere [5]. 


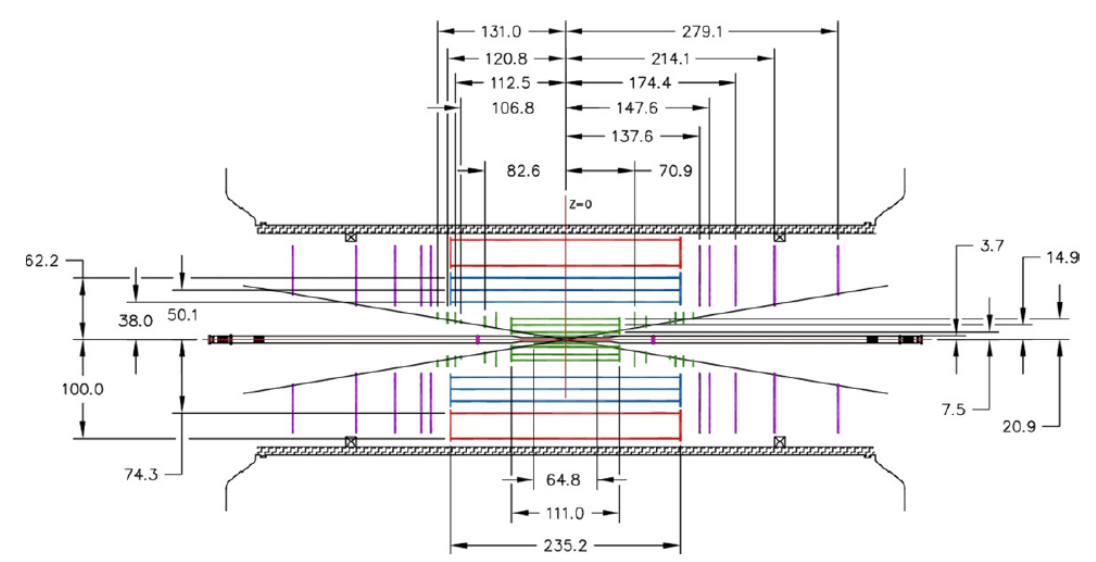

Figure 1: Layout of the proposed ATLAS Upgrade silicon tracker (from [3]). Pixel detector (green), short strip barrel (blue), long strip barrel (red), and strip endcaps (purple).

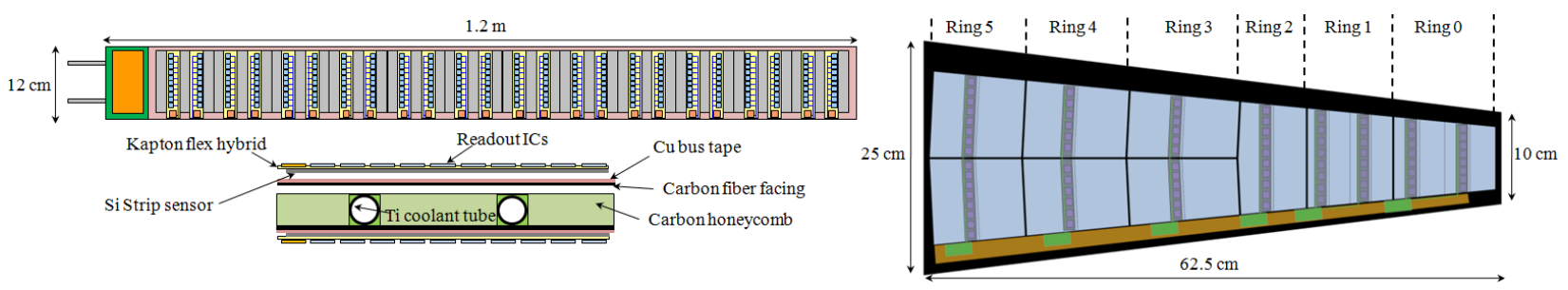

Figure 2: (Left) sketch of a barrel short strip stave. (Right) sketch of an end-cap petal.

\section{Barrel integration structures: staves}

\subsection{Stave module prototypes}

Each stave gives support to 24 sensor modules in total, 12 per side. Prototyping studies focused from the start in the production and test of short strip modules, which are the most challenging in the barrel region: in comparison to long strip modules, they host 4 times larger amount of readout channels (strips), and consequently they also dissipate higher power. According to the current strips tracker layout, almost 5200 short strip modules will be installed.

The n-in-p float zone (FZ) technology has been selected for the silicon micro-strip sensors of the upgraded tracker. It consists of a segmented $n^{+}$implant on a p-bulk FZ technology. The ATLAS collaboration, in collaboration with Hamamatsu Photonics, Ltd. has developed $9.75 \times 9.75 \mathrm{~cm}^{2}$ micro-strip sensors for the short strips region of the tracker, segmented in 4 columns of 1280 strips, each $2.39 \mathrm{~cm}$ long, and a pitch between strips equal to $74.5 \mu \mathrm{m}$. Two of those columns include axial strips, while the other two include stereo strips with a $40 \mathrm{mrad}$ angle. Each short strip sensor has in total 5120 strips, and the devices are $310 \mu \mathrm{m}$ thick. More details about the sensor design can be found in [6].

There are two hybrids in a short strip module, hosting the readout and control electronics necessary for the readout of the 5120 channels. Each hybrid includes 10 ABCN-13 ASICs, each with 256 channels and designed for binary readout, a Hybrid Controller Chip (HCC), plus some passive elements and part of the power circuitry. The ABCN-13 and the HCC ASICs will be fabricated in $130 \mathrm{~nm}$ CMOS process. This is still a conceptual idea, since the final designs of the ABCN-13 and HCC ASICs have not yet been finished. Current prototypes use the Basic Control Chips (BCCs), a short-term solution to fill in for the missing $\mathrm{HCC}$, and the $0.25 \mu \mathrm{m}$ CMOS version of the ABCNs, (ABCN-25), each with 128 channels. For that reason, prototypes built so far contain $20 \mathrm{ABCN}-25$ ASICs per hybrid instead of 10 .

Two power architectures have been considered for the staves: serial powering and DC-DC powering. In serial powering, a constant current source provides power to all the modules of a stave side with a shunt regulator circuit and a single power line. The current is constant along the chain, and is equal to the current required by an individual hybrid or module. There are as many voltage steps as elements in the chain. Another approach is used in DC-DC powering, in which a constant voltage source provides power to the modules in a stave side after one or several 
voltage conversion steps made by buck DC-DC converters. It also requires a single power line. The current increases along the stave, and the total current at the end of the stave is equal to the number of modules in the stave times the current required per hybrid, divided by the voltage conversion ratio. Strong R\&D efforts have been dedicated by the collaboration to the study and development of both powering architectures, each with advantages and drawbacks. No final decision has been taken yet. More details about both power distributions can be found in [7-11].

\subsection{Construction of stave module prototypes}

Almost 17000 strip modules will have to be assembled and installed in the silicon strip tracker. For that reason, module production has been designed with large scale requirements even at a prototyping stage. The hybrids have been designed in a kapton flex technology with no laminated stiffener material, in order to have the minimum amount of material. A 5 metal-layer panelized design has been selected for the hybrids [3]. This design allows machine placement of the passive components of the hybrids and solder reflow. Each panel hosts 8 hybrids, and conservative design rules have been followed in order to have high yield at low prize, such as $100 \mu \mathrm{m}$ track and gap, blind vias with $375 \mu \mathrm{m}$ lands with $150 \mu \mathrm{m}$ drill, and $50 \mu \mathrm{m}$ dielectrics. The panels are fabricated at Stevenage Circuits, Ltd. (UK). The hybrids are selectively laminated to a FR4 carrier, acting as a temporary substrate during assembly, wire bonding and test. Figure 3 shows one of these panels. Diverse tools have been designed for the controlled gluing and automated wire bonding of the ABCN ASICs to the panelized hybrids. This design allows electrical test of fully populated hybrids in the panels, prior to removal and module assembly. More than 80 fully functional hybrids have been produced so far by the collaboration, in different construction sites, with no failures [12].

Once the hybrids have been fabricated and tested, they are removed from the panels and glued directly onto the silicon strip sensors. The deposition of glue epoxy directly onto the strip sensors is a delicate process, and for that reason the process required to be properly addressed. With that purpose, miniature $\left(1 \times 1 \mathrm{~cm}^{2}\right)$, strip sensors, fabricated in the same wafers as the $9.75 \times 9.75 \mathrm{~cm}^{2}$ sensors, were prepared with different glues within the sensitive region of the sensor. It was found that when semiconductor grade epoxy Epolite FH-5313 was used, all miniature sensors held breakdown voltages well over $600 \mathrm{~V}$ after glue application and curing, and even after proton irradiations at fluences up to $1.5 \times 10^{15} n_{e q} / \mathrm{cm}^{2}$ [3]. The collaboration has also developed numerous tools for the uniform gluing of the hybrids to the sensors. Several options were investigated, such as different glues, spreading the glue directly onto the sensors or on the backplane of the hybrids, and different varieties of glue stencils. It was found that the optimized process for the best electrical performances of the modules requires the use of Epolite FH-5313 epoxy, and a glue thickness of $120 \mu \mathrm{m}$ [13]. Automated wire bonding machines have been also used for the wire bonding of the sensor short strips to the readout channels of the ABCN-25 ASICs in the hybrids. Automated procedures to bond two rows of 64 channels per ASIC (128 channels in total) have been developed.
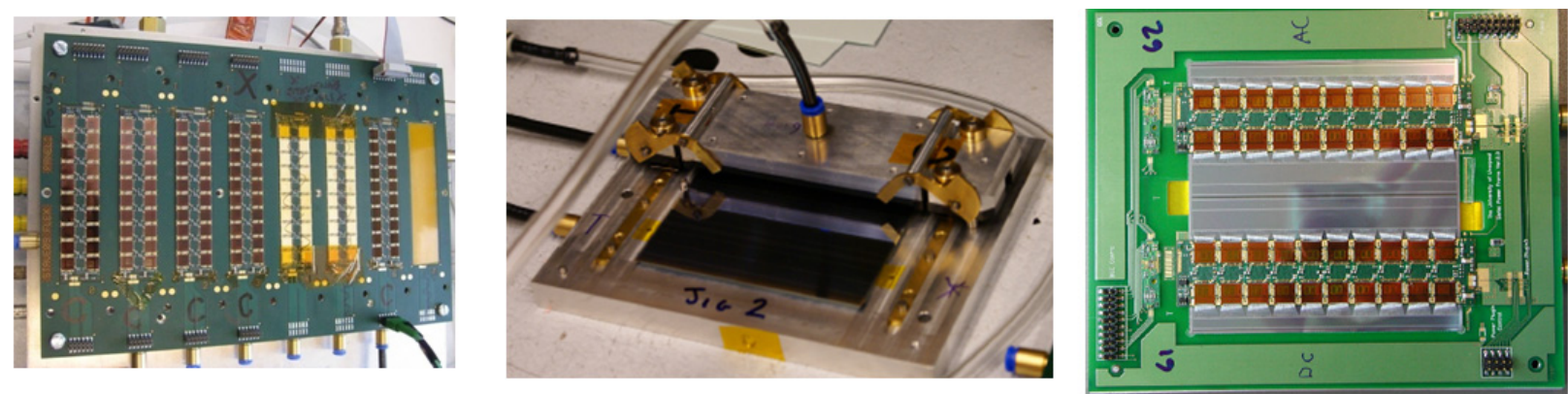

Figure 3: (Left) Panelized hybrids; (Middle) Gluing of hybrid onto sensor; (Right) Short strip module on PCB frame ready for test.

The stave modules are finally placed in removable Printed Circuit Board (PCB) frames that provide the traces and connections between the hybrids, power supplies and DAQ system, and the placement and connection of the BCC boards. The PCB testing frames allow the test of the modules with different powering distributions, grounding and shielding configurations, added circuitry, etc. The final result is a fully assembled and testable stave module, as shown in figure 3. Up to 31 short strip modules have been assembled, bonded, and tested with no failures by the different institutes of the collaboration [12]. 


\subsection{Electrical tests and irradiations}

As described in section 2.2, the production of short strip modules has been designed to have fully testable objects at the different stages of the building process. Short strip sensors, readout ASICs, hybrids, and module prototypes have been extensively tested by the collaboration, and several irradiation programs have been developed in order to check the radiation tolerance of the different components.

An extensive test program evaluated 20 full-size short strip sensors prior to irradiation. Results showed that the sensors were within the technical specifications with respect to leakage current $(<370 n \mathrm{~A}$ at $600 \mathrm{~V})$, interstrip capacitance and resistance $(0.8 \mathrm{pF} / \mathrm{cm}$ at $100 \mathrm{kHz}$ and $>150 \mathrm{G} \Omega / \mathrm{cm}$, respectively), coupling capacitance $(>28 \mathrm{pF} / \mathrm{cm}$ at $1 \mathrm{kHZ})$, and full depletion voltage $(<245 \mathrm{~V})$ [14]. Proton, pion, and neutron irradiation experiments were performed on miniature strip sensors up to $1.2 \times 10^{15} \mathrm{n}_{e q} / \mathrm{cm}^{2}$, the maximum fluence expected (including safety factors) at the innermost strip barrel layer. It was observed that the signal to noise ratio was greater than 10 at $500 \mathrm{~V}$ depletion voltage in all cases, with the expected noise performances $\left(\sim 600-800 e^{-}\right)[2]$.

Electrical test of hybrids and modules requires the use of highly multiplexed signals at high frequencies. The baseline DAQ system designed for that purpose is the High Speed Input/Output (HSIO) board [15]. It consists of a generic DAQ board with a single Virtex-4 Field Programmable Gate Array (FPGA) that allows data processing and connection to a controller PC. An additional interface board is required to host the connectors and buffers for the connectivity to the readout electronics. Additionally, a BCC ASIC per hybrid is required to provide AC-coupled Low Voltage Differential Signals (LVDS) clock and command, and to generate the $80 \mathrm{MHz}$ clock from the common $40 \mathrm{MHz}$ LVDS clock. Up to 64 simultaneous data streams are supported with this system. The system also uses an upgraded version of the sctdaq software, used in the past for the test of the current Semiconductor Tracker (SCT) modules.

All hybrids used in the construction of stave modules were electrically tested on panels independently prior to module assembly. Very similar results were obtained at the different construction sites: the performance of the ASICs on the hybrids matches bare chip studies, the average noise per channel on single hybrids measured is $\sim 400 e^{-}$, the gain is equal to $100 \mathrm{mV} / \mathrm{fC}$, and the threshold variation is $5.5 \mathrm{mV}$ before trimming and $1 \mathrm{mV}$ after trimming [3]. The electrical performances of the stave modules depends strongly on the powering scheme used (parallel, serial, or DC-DC). However, it was found that, with appropriate grounding and shielding configurations, electrical performance of stave modules using parallel, serial, and DC-DC powering has shown to be the same, showing values of average noise per channel of $\sim 600 e^{-}$with the sensors biased at $200 \mathrm{~V}$ in all cases [13]. The noise performance of the stave modules has been reproduced systematically with the modules built and tested at different sites (and with different power distributions) within the collaboration. Figure 4 shows the output noise from one of the columns of one single hybrid tested in a panel at Berkeley Lab. It also shows the output noise of the same column after its assembly into a fully functional serial powered stave module, with the sensor biased at $200 \mathrm{~V}$.
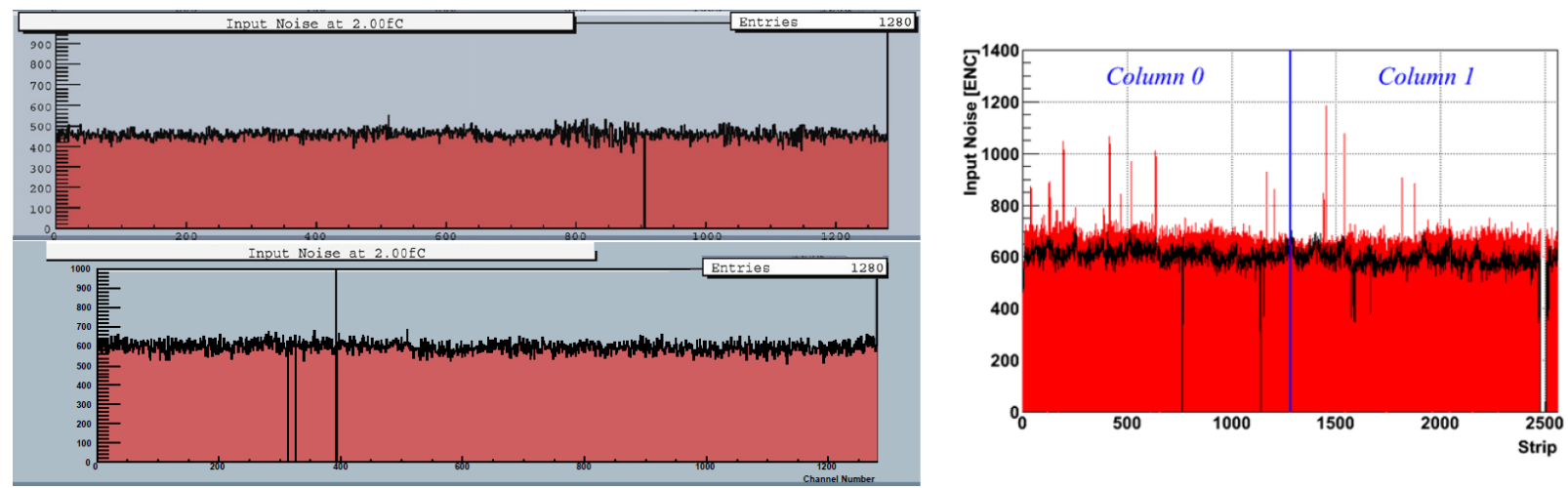

Figure 4: (Left top) Output noise of a chip column of a short strip hybrid tested on a panel, prior to assembly; (Left bottom) Output noise of the same hybrid column after module assembly. (Right) Output noise of a short strip hybrid after $2 \times 10^{15} n_{e q} / \mathrm{cm}^{2}$ proton irradiation (from [12]). (Black) Pre-irrad values. (Red) Post-irrad values.

Additionally, in order to check the radiation tolerance of the stave modules, proton irradiation tests were performed 
with two stave modules at CERN PS irradiation facility [16]. The fluence achieved during the irradiation was equal to $2 \times 10^{15} n_{e q} / \mathrm{cm}^{2}$, which exceeds the maximum fluence expected in the strips tracker. During irradiation, sensors were biased to $100 \mathrm{~V}$ and the readout ASICs were powered and clocked. The sensor leakage currents were measured at $500 \mathrm{~V}$ after irradiation without the ASICs powered, showing to be consistent with expectations for the given fluence and sensor temperatures. The average noise per channel was evaluated before and after irradiation at $200 \mathrm{~V}$. Figure 4 shows the results for two columns of 10 chips of one of the modules. The average $70 e^{-}$noise increase observed after irradiation is consistent with shot noise expectations for the measured reverse current and the shaping time of the ASICs [12].

\subsection{Stavelet prototypes}

Multi-module prototypes of short strips, the so-called 'stavelets' have been designed and constructed: they consist of single-sided stave prototypes with 4 short strip modules, in which the modules are directly glued to a co-cured copper bus tape with 'soft' glue for easy module replacement or removal. The edges of the tapes have been widened to provide space to attach the power and protection components on one edge, and the BCC boards on the other. Those prototypes have proved to be very powerful test beds for electrical testing, such as the different powering distributions, grounding and shielding configurations, etc. Two stavelets have been built so far: one with serial powering, and the other with DC-DC powering and a parallel power bus tape. Figure 5 shows a picture of both stavelets. The first results reveal very a similar electrical behavior for both stavelets, showing an overall output noise increase of approximately $20 e^{-}$of the modules with respect to their measurement in individual module test frames [7, 17].
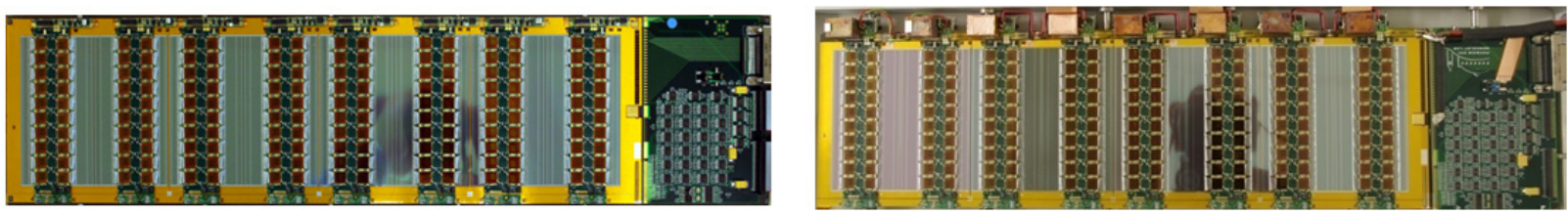

Figure 5: (Left) Serially powered stavelet. (Right) DC-DC powered stavelet.

\section{Endcap integration structures: petals}

The end-cap petals follow very closely the design of the barrel staves. However, the design of the petal is more complex in comparison to the barrel. Each petal hosts 9 sensor modules per side (18 modules per petal in total). The modules are not identical within each petal: there are in total 6 different types of sensors, and 8 different types of hybrids. The petals can be divided in 6 different regions, or rings, depending on the type of sensor each ring hosts, as detailed in figure 2 [18].

Due to this increased complexity, the prototyping stage of the petals is not as mature as in the case of the barrel modules. A first prototype of a petal module, the so-called 'petalet', has already been designed [19]. The petalet combines the two regions of the petals that may reveal specific testing issues not present in the barrel prototypes: the innermost radius sensors, and the region in which the petal splits into two sensor columns. Figure 6 shows a sketch of the petalet prototype. Petalet sensors and hybrids have been designed and are currently under construction [20]. First electrical results from the petalet are expected by the end of 2012.

\section{Conclusions}

The stave program, a collaboration effort to build the ATLAS Upgrade silicon strip tracker involving more than 20 institutes worldwide, has shown significant progress in the last year. Up to 31 stave module prototypes have been built and shown to work after irradiation at higher fluences than expected on the Si tracker. In addition, two multi-module stavelet prototypes have been built and are currently under test. A significant improvement has been also shown in the end-cap module prototypes, with a 'petalet' expected to be built and tested in the following months. Existing and forthcoming prototypes constitute powerful tools for the collaboration for a deeper study of some of the key aspects 


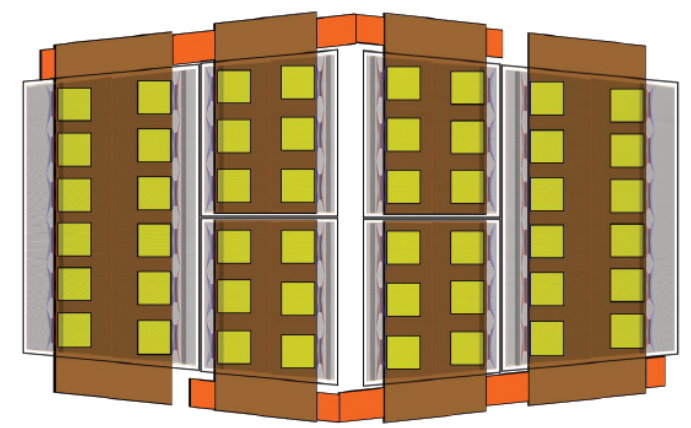

Figure 6: Sketch of a petalet (from [19]).

of the detector, such as the power distribution, or the grounding and shielding strategies. The design of full-size, next generation prototypes has already started, and they will be built as soon as the ABCN-130 and HCC ASICs are ready, also during 2012.

\section{Acknowledgments}

Authors want to acknowledge the staff and team of the irradiation facilities at PSI (Villigen), JSI (Ljubljana), CERN (Geneva), University of Karlsruhe, and Univedrsity of Tohoku, for their efficiency and dedication in performing the irradiation of the silicon strip prototypes.

\section{References}

[1] F. Gianotti et al., Eur. Phys. J. C 39 (2005) 293-333.

[2] H. F.-W. Sadrozinski et al., Nucl. Inst. Meth. A 658 (2011) 20 - 24.

[3] P. P. Allport et al., Nucl. Inst. Meth. A 636 (2011) S90 - S96.

[4] ATLAS Inner Tracker Steering Committee, Silicon strip tracker, 2012. Backup document of the Letter of Intent for the Phase-II Upgrade of the ATLAS Experiment, to be published.

[5] S. Gonzalez-Sevilla et al., ATL-COM-UPGRADE-2011-021 (2011). http://cdsweb.cern.ch/record/1387541.

[6] Y. Unno et al., Nucl. Inst. Meth. A 636 (2011) S24 - S30.

[7] J. Matheson, J. Inst. 6 (2011) C01019.

[8] D. Lynn et al., Nucl. Inst. Meth. A 633 (2011) $51-60$

[9] B. Allongue et al., J. Inst. 5 (2010) C11011.

[10] F. Faccio et al., J. Inst. 5 (2010) C11016.

[11] S. Diez, System implications of the different powering distributions for the ATLAS Upgrade strips tracker, 2012. Physics Procedia, in press.

[12] A. Affolder, Silicon strip detectors for the ATLAS HL-Upgrade, 2012. Physics Procedia, in press.

[13] A. Affolder, in: ACES 2011 Workshop. https://indico.cern.ch/conferenceOtherViews.py?view=standard\&confId=113796.

[14] J. Bohm et al., Nucl. Inst. Meth. A 636 (2011) S104 - S110.

[15] D. Nelson, HSIO Development Users guide, 2010. Available online at: http://www.slac.stanford.edu/ djn/Atlas/hsio/.

[16] M. Glaser et al., Nucl. Inst. Meth. A 426 (1999) 72-77.

[17] P. Phillips, in: TWEPP 2011 Workshop. https://indico.cern.ch/conferenceDisplay.py?ovw=True\&confId=120853.

[18] C. Lacasta, in: AUW Nov 2010. https://indico.cern.ch/conferenceDisplay.py?confId=75718.

[19] C. Lacasta, in: AUW Nov 2011. https://indico.cern.ch/conferenceDisplay.py?confId=108365.

[20] V. Benitez et al., in: AUW Nov 2011. https://indico.cern.ch/conferenceDisplay.py?confId=108365. 